\title{
CUSTO DIRETO DA HEMODIÁLISE EM UNIDADE DE TERAPIA INTENSIVA ADULTO
}

\author{
Jony Hsiao ${ }^{1}$, Antônio Fernandes Costa Lima²
}

${ }^{1}$ Bacharel em Ciências Contábeis. Mestre em Ciências. Graduando em Enfermagem. Escola de Enfermagem da Universidade de São Paulo. São Paulo, SP, Brasil.

${ }^{2}$ Enfermeiro. Doutor em Enfermagem. Docente do Departamento de Orientação Profissional da Escola de Enfermagem da Universidade de São Paulo. São Paulo, SP, Brasil.

RESUMO: Objetivou-se identificar o custo direto médio total da hemodiálise convencional realizada por técnicos de enfermagem a pacientes com lesão renal aguda em Unidade de Terapia Intensiva Adulto. Pesquisa quantitativa em estudo de caso, realizada em hospital público de ensino e pesquisa, entre janeiro e abril de 2014. Calculou-se o custo direto médio das etapas constituintes da hemodiálise multiplicando-se o tempo despendido na sua execução pelo custo unitário da mão-de-obra direta, somando-se ao custo dos materiais, soluções e medicamentos. Obteve-se o custo direto médio total de $\mathrm{R} \$ 434,83$ ( $\mathrm{DP}=65,10$ ) que foi impactado pelos custos diretos médios das etapas "monitorização" (R\$ 205,58), "preparo da máquina e do circuito extracorpóreo" (R\$ 120,96) e "desinfecção interna e limpeza da máquina" (R\$ 42,10). Conclui-se que a metodologia proposta poderá ser reproduzida em outras Unidades de Terapia Intensiva subsidiando a tomada de decisões com vistas à eficiência alocativa dos recursos envolvidos na consecução da hemodiálise convencional.

DESCRITORES: Lesão renal aguda; Unidades de terapia intensiva; Unidades hospitalares de hemodiálise; Enfermagem em nefrologia; Custos e análise de custo.

\section{DIRECT COST OF HEMODIALYSIS IN AN ADULT INTENSIVE CARE UNIT}

\begin{abstract}
The present study aimed to identify the average direct cost of conventional hemodialysis performed by nursing technicians on patients with acute kidney injury in Adult Intensive Care Unit. Quantitative analysis in case study research conducted in a public teaching and research hospital, between January and April 2014. The average direct cost of the stages of hemodialysis was calculated by multiplying the time needed to perform the procedure by the unit cost of direct labor and adding this value to the cost of materials, solutions and drugs. The total direct cost obtained was $\mathrm{R} \$ 434,83$ (SD=65.10), which was impacted by the average direct costs of the "monitoring" steps" (R\$205,58), "preparation of the machine and extracorporeal circuit" (R\$ 120,96) and "internal disinfection and machine cleaning" ( $R \$ 42,10)$. It is concluded that the proposed methodology can be reproduced in other Intensive Care Units to assist in the decision making regarding allocative efficiency of the resources involved in the procedure of conventional.

DESCRIPTORS: Acute kidney injury; Intensive care units; Hemodialysis hospital units; Nephrology nursing; Costs and cost analysis.
\end{abstract}

\section{COSTO DIRECTO DE HEMODIÁLISIS EN UNIDAD DE TERAPIA INTENSIVA ADULTO}

RESUMEN: Estudio cuya finalidad fue identificar el costo directo medio total de la hemodiálisis convencional realizada por técnicos de enfermería a pacientes con lesión renal aguda en Unidad de Terapia Intensiva Adulto. Investigación cuantitativa en estudio de caso, realizada en hospital público de enseñanza e investigación, entre enero y abril de 2014. Se calculó el costo directo medio de las etapas de la hemodiálisis multiplicándose el tiempo empleado en su ejecución por el costo unitario de la mano de obra directa, sumándose al costo de los materiales, soluciones y medicamentos. Se obtuvo el costo directo medio total de $\mathrm{R} \$ 434,83(\mathrm{DP}=65,10)$ que fue impactado por los costos directos medios de las etapas "monitorización" ( $\mathrm{R} \$ 205,58)$, "preparo de la máquina y del circuito extracorpóreo" (R\$ 120,96) y "desinfección interna y limpieza de la máquina" $(R \$ 42,10)$. Se concluye que la metodología propuesta podrá ser reproducida en otras Unidades de Terapia Intensiva subsidiando las decisiones para llegar a la eficiencia alocativa de los recursos presentes en la consecución de la hemodiálisis convencional.

DESCRIPTORES: Lesión renal aguda; Unidades de terapia intensiva; Unidades hospitalares de hemodiálisis; Enfermería en nefrología; Costos y análisis de costo. 


\section{INTRODUÇÃO}

No Brasil, o quantitativo de pacientes dependentes de terapia renal substitutiva (TRS) aumenta a cada ano, ocasionando preocupações crescentes para os gestores do Sistema Único de Saúde (SUS) em relação à sua eficiência e seus custos, visto abranger procedimentos de alta complexidade e, consequentemente, de alto custo $^{(1)}$. Tal realidade também é percebida em outros países que têm enfrentado o desafio de gerenciar altos custos para a manutenção dos pacientes em diferentes modalidades dialíticas ${ }^{(2-4)}$.

Os pacientes críticos internados em Unidade de Terapia Intensiva (UTI), que desenvolvem lesão renal aguda (LRA), necessitam de alguma modalidade de TRS, intermitente (hemodiálise convencional - HDC ou diálise peritoneal) ou contínua (hemodiálise, hemofiltração ou hemodiafiltração)(5), cuja indicação dependerá do seu estado clínico, do conhecimento médico e da disponibilidade de recursos ${ }^{(6)}$.

Historicamente, a HDC tem sido a opção preferencial de tratamento para os pacientes dependentes de $\operatorname{TRS}^{(7)}$. Tal modalidade dialítica requer a obtenção e manutenção de uma via de acesso à circulação sanguínea; a utilização de soluções, materiais e equipamentos específicos; e a disponibilidade de profissionais especializados e de recursos estruturais que contemplem as Resoluções e Portarias estabelecidas pelo Ministério da Saúde $(\mathrm{MS})^{(8)}$.

De acordo com as normas legais vigentes no cenário nacional, os membros da equipe de saúde (médico nefrologista, enfermeira, nutricionista, psicóloga, assistentesocial, terapeutaocupacional, técnicos de enfermagem) devem permanecer no Centro de Diálise (CD), durante toda a sessão, assumindo a responsabilidade pelo atendimento no procedimento hemodialítico ${ }^{(9)}$ e, apesar de não ser prerrogativa exclusiva dos profissionais de enfermagem, estes estão presentes, o tempo todo, nos períodos pré, trans e pós-diálise.

Nas instituições hospitalares, a HDC, destinada aos pacientes portadores de LRA em condições críticas, é geralmente realizada por enfermeiros ou técnicos de enfermagem atuantes em seu CD que se deslocam até a UTI. A participação de enfermeiros ou técnicos de enfermagem é condicionada e determinada pela capacidade de atendimento e área física do $\mathrm{CD}$; relação entre o quantitativo de profissionais de enfermagem, por categoria, bem como pelo quantitativo e condições clínicas dos pacientes atendidos. $\mathrm{Na}$ grande maioria das vezes, a HDC em UTI é realizada por técnicos de enfermagem, devidamente capacitados e altamente qualificados, sob a supervisão à distância do médico nefrologista e da enfermeira do CD.

O processo decisório em relação aos recursos humanos, materiais, estruturais e econômicos, necessários à viabilização da HDC, pode ser continuamente melhorado a partir do conhecimento de como se comportam os custos das etapas que a compõe. Então, a apuração dos custos dos insumos consumidos, neste procedimento dialítico, é estratégia fundamental para instrumentalizar a sua alocação eficiente e favorecer a comparação com o valor atualmente repassado pelo SUS de $\mathrm{R} \$ 261,41$ por sessão de HDC de pacientes renais agudos/crônicos agudizados, sem tratamento dialítico iniciado ${ }^{(10)}$.

Destaca-se que a possibilidade de comparação de valores, incorridos e financiados, propiciaria às organizações de saúde, especialmente aos hospitais públicos de ensino e pesquisa, a elaboração de argumentações diante da necessidade de ajustes, pois desde 2004 passaram a firmar um contrato com o gestor (União, Estados, Distrito Federal, Municípios), estabelecendo metas e indicadores de resultado a serem cumpridos, assim como o repasse dos recursos financeiros ${ }^{(11)}$.

Pesquisas relacionadas ao gerenciamento de custos das diferentes modalidades de TRS ainda são escassas, principalmente no Brasil. Destarte, investimentos na aplicação de metodologias para a apuração dos custos dos procedimentos hemodialíticos precisam ser realizados, a fim de fundamentar avaliações de sustentabilidade econômica, sem prejuízos à qualidade assistencial.

A realização de estudos desta natureza em hospitais públicos de ensino e pesquisa tornase imperativa, pois o conhecimento dos custos dos serviços, das ações assistenciais agregadas às atividades de ensino e pesquisa condicionam e determinam $\mathrm{o}$ aumento de $\operatorname{custos}^{(12)}$. Nesta perspectiva, torna-se fundamental o envolvimento dos enfermeiros nas mudanças dos processos de trabalho com vistas ao alcance de melhores resultados, utilização racional dos recursos e otimização dos custos, colaborando com a sustentabilidade financeira das instituições de saúde.

Frente ao exposto, objetivou-se identificar o custo direto médio total (CDMT) da HDC realizada por técnicos de enfermagem a pacientes portadores de LRA internados em Unidade de 
Terapia Intensiva Adulto (UTI-A).

\section{MÉTODO}

Trata-se de pesquisa quantitativa, exploratóriodescritiva, do tipo estudo de caso único, método que possibilita a compreensão de um fenômeno contemporâneo da vida real, considerando as condições contextuais para responder questões do tipo "como" e "por quê" em uma situação na qual o pesquisador "tem pouco controle" sobre os acontecimentos ${ }^{(13)}$.

O estudo foi realizado em um hospital público de ensino e pesquisa após a aprovação dos Comitês de Ética e Pesquisa da instituição proponente (Parecer $\mathrm{n}^{\circ}$ : 489.961) e do hospital campo de estudo - HCE (Parecer $n^{\circ}$ : 492.808).

Devido à imprevisibilidade da ocorrência de LRA em pacientes críticos e da variabilidade da indicação da HDC como modalidade dialítica de escolha para seu tratamento, estabeleceu-se uma amostra de conveniência. Assim, a coleta de dados ocorreu no período de 26 de janeiro a 26 de abril de 2014 visando a obtenção do maior número possível de observações do procedimento em estudo. Neste período, 23 pacientes internados na UTI-A apresentaram LRA e todos compuseram a amostra.

O HCE, selecionado por apresentar boas práticas de enfermagem, adequação estrutural, tecnológica e de recursos humanos, é um órgão complementar de uma Universidade Pública, mantida pelo Estado de São Paulo e ligada à Secretaria de Estado de Desenvolvimento Econômico, Ciência e Tecnologia.

A UTI-A dispõe de 12 leitos destinados aos cuidados intensivos e oito aos cuidados semiintensivos para o atendimento de pacientes com idade superior a 15 anos, sendo a maioria idosa, portadora de doenças cônicas agudizadas, provenientes de diversas unidades do HCE e também de outras instituições de saúde.

O CD atende a pacientes portadores de doença renal crônica terminal (DRCT) e LRA, de segunda-feira a sábado, em dois turnos: das 7 às 13 horas e das 13 às 19 horas. A equipe de enfermagem é composta por três enfermeiras e quatro técnicos distribuídos de forma a garantir, no mínimo, uma enfermeira e dois técnicos por turno. Estruturalmente, está organizado para dialisar seis pacientes por período, sendo quatro pacientes com DRCT e dois pacientes com LRA.

Diariamente os médicos nefrologistas avaliam os pacientes com risco de desenvolverem LRA internados no $\mathrm{HCE}, \mathrm{e}$, caso haja a indicação de HDC, esta é programada no horário de funcionamento do CD. Nos casos de pacientes internados na UTI-A, considerados pelos médicos nefrologista e intensivista como em condições clínicas críticas que inviabilizem o seu encaminhamento ao $C D$, a enfermeira do $C D$ escala um técnico de enfermagem para realizar a HDC no leito onde o mesmo está internado, com supervisão à distância. Para tanto, utiliza-se uma máquina de hemodiálise exclusiva, diferenciada das demais, e um carro de transporte contendo os materiais, soluções e medicamentos específicos.

Para a apuração dos custos relativos ao uso de materiais, soluções, medicamentos e mão-deobra especializada, foram utilizados os custos diretos. Custos diretos são definidos como um sacrifício monetário que se aplica na geração de um produto ou de um serviço em que há possibilidade de identificação com o produto ou departamento, passível de ser identificado, mensurado e claramente quantificado ${ }^{(14)}$.

O custo unitário da mão-de-obra direta (MOD) dos técnicos de enfermagem do CD foi calculado a partir dos salários médios, relativos ao período de coleta de dados, fornecidos pelo Diretor Financeiro do Hospital. A MOD referese ao pessoal que trabalha, diretamente, sobre um produto ou serviço prestado, desde que seja possível mensurar o tempo despendido e identificar quem executou o trabalho. Compõese dos salários, encargos sociais, provisões para férias e $13^{\circ}$ salário $^{(14)}$.

O responsável pelo Almoxarifado do HCE forneceu os valores relativos à última aquisição de materiais, soluções e medicamentos previstos em cota do CD.

Para favorecera rápida documentação do tempo (cronometrado) despendido pelos técnicos de enfermagem e do consumo de materiais, soluções e medicamentos, sem prejuízos à condução das seções de HDC na UTI-A, foi construído, testado e validado um instrumento, junto aos enfermeiros e técnicos do CD.

A primeira parte do instrumento destinava-se ao registro da data, turno, número de prontuário do paciente, e a segunda, estruturada em sete etapas, ao registro do tempo de duração, quantidade de materiais, soluções e medicamentos consumidos e observações. As etapas e os insumos da segunda parte do instrumento são apresentados no Quadro 1. 
Quadro 1 - Etapas constituintes do procedimento hemodiálise convencional e respectivos insumos - São Paulo, SP, Brasil, 2014

\begin{tabular}{|c|c|c|}
\hline Etapa & \multicolumn{2}{|c|}{ Insumos } \\
\hline $\begin{array}{l}1^{\mathrm{a}} \text { - Preparo da máquina e } \\
\text { do circuito extracorpóreo }\end{array}$ & $\begin{array}{l}\text { Técnico de Enfermagem do CD } \\
\text { Dialisador capilar } \\
\text { Linhas de sangue arterial } \\
\text { Linha de sangue venosa } \\
\text { Frasco de SF } 0,9 \% \text { - } 1000 \mathrm{ml} \\
\text { Equipo de soro } \\
\text { Isolador do condutor de pressão }\end{array}$ & $\begin{array}{l}\text { Luvas de procedimento } \\
\text { Frasco de reagente para peróxido de } \\
\text { hidrogênio } \\
\text { Bicarbonato de Sódio 8,4W\% (galão - } \\
5 \text { litros) } \\
\text { Solução Polieletrolítica (galão - } 5 \text { litros) }\end{array}$ \\
\hline $2^{\mathrm{a}}$ - Instalação & $\begin{array}{l}\text { Técnico de Enfermagem do CD } \\
\text { Luvas estéreis } \\
\text { Avental de manga longa } \\
\text { Protetor facial } \\
\text { Luvas de procedimentos não estéreis } \\
\text { Máscaras } \\
\text { Seringa de } 20 \mathrm{ml} \\
\text { Seringa de } 10 \mathrm{ml} \\
\text { Seringa de } 03 \mathrm{ml} \\
\text { Clorexidina alcoólica ( } 100 \mathrm{ml}) \\
\text { Campo fenestrado estéril }\end{array}$ & $\begin{array}{l}\text { Pacote de gaze estéril } \\
\text { Fita micropore de } 50 \mathrm{~mm}(100 \mathrm{~cm}) \\
\text { Fita micropore de } 100 \mathrm{~mm}(30 \mathrm{~cm}) \\
\text { Pacote de curativo cirúrgico } \\
\text { Bandeja de anti-sepsia } \\
\text { Frasco de SF - } 10 \mathrm{ml} \\
\text { Frasco-ampola de heparina } \\
\text { Pomada de Mupirocina }(1,2 \text { gramas }) \\
\text { Agulhas } 30 x 8 \\
\text { Conectores TEGO }\end{array}$ \\
\hline $\begin{array}{l}3^{\mathrm{a}} \text { - Administração de } \\
\text { soluções/medicamentos } \\
\text { durante a HDC }\end{array}$ & $\begin{array}{l}\text { Frasco de } \mathrm{SF} 0,9 \%-1000 \mathrm{ml} \\
\text { Cloreto de potássio } \\
\text { Seringa de } 20 \mathrm{ml} \\
\text { Seringa de } 10 \mathrm{ml}\end{array}$ & $\begin{array}{l}\text { Seringa de } 03 \mathrm{ml} \\
\text { Agulha } 30 \times 7 \\
\text { Bola de algodão com álcool }\end{array}$ \\
\hline $4^{\mathrm{a}}-$ Desinstalação & $\begin{array}{l}\text { Técnico de Enfermagem do CD } \\
\text { Luvas estéreis } \\
\text { Avental de manga longa }\end{array}$ & $\begin{array}{l}\text { Protetor facial } \\
\text { Máscara } \\
\text { Frasco-ampola de heparina }(\mathrm{ml})\end{array}$ \\
\hline $5^{\mathrm{a}}$ - Monitorização & \multicolumn{2}{|l|}{ Técnico de Enfermagem do CD } \\
\hline $\begin{array}{l}6^{\mathrm{a}} \text { - Desinfecção interna e } \\
\text { limpeza externa da máquina }\end{array}$ & $\begin{array}{l}\text { Técnico de Enfermagem do CD } \\
\text { Ácido Peracético-Puresteril (300 ml) } \\
\end{array}$ & $\begin{array}{l}\text { Álcool a } 70 \%(50 \mathrm{ml}) \\
\text { Pano multiuso }\end{array}$ \\
\hline $\begin{array}{l}7^{\mathrm{a}}-\text { Tempo total de } \\
\text { deslocamento }\end{array}$ & \multicolumn{2}{|l|}{ Técnico de Enfermagem do CD } \\
\hline
\end{tabular}

$\mathrm{Na} 3^{\mathrm{a}}$ etapa "administração de soluções/ medicamentos durante a HDC", foram registradas apenas as soluções/medicamentos relacionados especificamente ao procedimento dialítico, portanto não foram contabilizados os custos de outros medicamentos em uso devido à internação do paciente na UTI-A. Nesta etapa foram excluídos o tempo de duração e o custo com a MOD do Técnico de Enfermagem do CD, pois se optou por considerar, essas variáveis, na $5^{\mathrm{a}}$ etapa "monitorização" para que fosse contabilizada uma única vez.

O custo direto médio (CDM) de cada etapa foi obtido multiplicando-se o tempo (cronometrado) despendido por técnicos de enfermagem pelo custo unitário da MOD, somando-se ao custo dos materiais, soluções e medicamentos utilizados. Para a realização dos cálculos, utilizou-se a moeda brasileira real $(\mathrm{R} \$)$. As variáveis numéricas foram analisadas, descritivamente, pelo número absoluto e relativo das respostas, e os valores apresentados em tabelas.

\section{RESULTADOS}

Foram documentadas 57 sessões de HDC destinadas a 23 pacientes com LRA, portadores de cateter de duplo lúmen (CDL) como via de acesso à circulação sanguínea, internados na UTI-A, nos períodos da manhã e da tarde. O número de sessões de HDC variou de uma a três, sendo mais frequentes três sessões $(78,3 \%)$ por paciente.

Dos técnicos de enfermagem, (75\%) executantes das sessões de HDC era do sexo feminino, com média de idade de 38,5 anos (Desvio Padrão - $\mathrm{DP}=6,81$ ) e tempo médio de atuação em hemodiálise de 10,25 ( $\mathrm{DP}=4,03)$. O salário médio mensal, por uma carga de trabalho de 144 horas, correspondeu a R\$7.249,07, sendo $\mathrm{R} \$ 50,34$ relativos à MOD/hora e $\mathrm{R} \$ 0,84$ a MOD/ minuto.

Na Tabela 1, demonstra-se o CDM das sete etapas integrantes do procedimento HDC conforme o tempo de duração, custos com 
Tabela 1 - Distribuição das etapas do procedimento hemodiálise convencional, segundo o tempo de duração e custos diretos com pessoal, material e soluções/medicamentos. São Paulo, SP, Brasil, 2014

\begin{tabular}{|c|c|c|c|c|c|c|}
\hline Etapas da HDC $¥$ & $\mathbf{n}$ & Média & DP & Mediana & Mín-Máx & Moda \\
\hline \multicolumn{7}{|c|}{$1^{\text {a }}$ Preparo da máquina e do circuito extracorpóreo } \\
\hline Duração (minutos) & 57 & 16,95 & 4,82 & 15 & Oct-30 & 15 \\
\hline Custo com pessoal & 57 & 14,24 & 4,05 & 12,6 & $8,40-25,20$ & 12,6 \\
\hline Custo com material & 57 & 84,04 & 19,17 & 82,4 & $2,18-166,49$ & 82,4 \\
\hline Custo com soluções/medicamentos & 57 & 22,68 & 6,24 & 21 & $1,90-40,10$ & 21 \\
\hline CDM+ da $1^{\mathrm{a}}$ etapa & 57 & 120,96 & 20,29 & 116,84 & $54,88-209,39$ & 116 \\
\hline \multicolumn{7}{|l|}{$2^{\text {a } \text { Instalação }}$} \\
\hline Duração (minutos) & 57 & 8,11 & 3,77 & 6 & Apr-20 & 5 \\
\hline Custo com pessoal & 57 & 6,81 & 3,17 & 5,04 & $3,36-16,80$ & 4,2 \\
\hline Custo com material & 57 & 13,12 & 9,44 & 12,87 & $2,42-24,98$ & 3,05 \\
\hline Custo com soluções/medicamentos & 57 & 8,63 & 0,41 & 8,54 & $7,96-10,36$ & 8,54 \\
\hline CDM+ da $2^{a}$ etapa & 57 & 28,56 & 9,39 & 29,12 & $15,06-45,76$ & 19,99 \\
\hline \multicolumn{7}{|c|}{$3^{\text {a }}$ Administração de soluções/medicamentos } \\
\hline Custo com material & 57 & 1,71 & 0,86 & 2,07 & $0-3,80$ & 2,28 \\
\hline Custo com soluções/medicamentos & 57 & 9,08 & 0,99 & 8,54 & $7,96-13,29$ & 8,54 \\
\hline CDM+ da $3^{\mathrm{a}}$ etapa & 57 & 10,79 & 1,46 & 10,82 & $7,96-15,57$ & 10,82 \\
\hline \multicolumn{7}{|l|}{$4^{a}$ Desinstalação } \\
\hline Duração (minutos) & 57 & 8 & 2,82 & 8 & Apr-16 & 8 \\
\hline Custo com pessoal & 57 & 6,72 & 2,36 & 6,72 & $3,36-13,44$ & 6,72 \\
\hline Custo com material & 57 & 0,9 & 0,48 & 0,75 & $0-3,72$ & 0,75 \\
\hline Custo com soluções/medicamentos & 57 & 1,17 & 2,54 & 0 & $0-6,56$ & 0 \\
\hline CDM+ da $4^{\mathrm{a}}$ etapa & 57 & 8,79 & 2,98 & 7,8 & $1,08-17,16$ & 7,47 \\
\hline \multicolumn{7}{|l|}{$5^{\text {a }}$ Monitorização } \\
\hline Duração (em minutos) & 57 & 244,73 & 65,71 & 240 & $80-480$ & 240 \\
\hline CDM+ com pessoal na $5^{\mathrm{a}}$ etapa & 57 & 205,58 & 55,2 & 201,6 & $67,20-403,20$ & 201,6 \\
\hline \multicolumn{7}{|c|}{ 6 ${ }^{a}$ Desinfecção interna e limpeza externa máquina } \\
\hline Duração (minutos) & 57 & 39,57 & 8,47 & 40 & $20-70$ & 40 \\
\hline Custo com pessoal & 57 & 33,24 & 7,11 & 33,6 & $16,80-58,80$ & 33,6 \\
\hline Custo com material & 57 & 0,33 & 0,12 & 0,28 & $0,28-0,84$ & 0,28 \\
\hline Custo com soluções/medicamentos & 57 & 8,53 & 1,6 & 8,85 & $0-8,85$ & 8,85 \\
\hline CDM+ da $6^{\mathrm{a}}$ etapa & 57 & 42,1 & 7,41 & 42,73 & $21,85-68,21$ & 42,73 \\
\hline \multicolumn{7}{|l|}{$7^{\text {a }}$ Tempo total de Deslocamento } \\
\hline Duração (minutos) & 57 & 21,48 & 6,48 & 20 & Jun-35 & 20 \\
\hline CDM+ com pessoal na $7^{\mathrm{a}}$ etapa & 57 & 18,05 & 5,44 & 16,8 & $5,04-29,40$ & 16,8 \\
\hline CDMT§ do procedimento HDC $\ddagger$ & 57 & 434,83 & 65,1 & 426,7 & $308,93-741,48$ & - \\
\hline
\end{tabular}

pessoal, com material, soluções/medicamentos. O custo direto por seção de HDC variou entre $\mathrm{R} \$ 308,93$ e $\mathrm{R} \$ 741,48$, obtendo-se o custo direto médio total (CDMT) de $\mathrm{R} \$ 434,83$ ( $\mathrm{DP}=65,10)$, com tempo médio de duração de 338,84 minutos $(6,65$ horas).

As etapas "monitorização" e "desinfecção interna e limpeza externa da máquina" apresentaram o maior CDM com pessoal, R\$205,58 $(72,69 \%)$ e $\mathrm{R} \$ 33,24(11,75 \%)$; " preparo da máquina e do circuito extracorpóreo" e "instalação" o maior CDM com materiais, $\mathrm{R} \$ 84,04(88,77 \%)$ e $\mathrm{R} \$$ $13,12(13,86 \%)$ e "preparo da máquina e do circuito extracorpóreo" e "administração de soluções/ medicamentos durante a HDC" o maior CDM com soluções/medicamentos, $\mathrm{R} \$ 22,68$ (48,04\%) e $\mathrm{R} \$ 9,08(19,23 \%)$.

Verifica-se, na Tabela 2, o maior impacto do CDM das etapas "monitorização", R\$ 205,58 $(47,28 \%)$, "preparo da máquina e do circuito 
Tabela 2 - Distribuição dos custos diretos com pessoal, material e soluções/medicamentos segundo as etapas do procedimento hemodiálise convencional. São Paulo, SP, Brasil, 2014

\begin{tabular}{|c|c|c|c|c|c|}
\hline Etapas componentes da HDC†+ & $\begin{array}{c}\text { CDM } ¥ \\
\text { com pessoal } \\
\text { R\$ }\end{array}$ & $\begin{array}{c}\text { CDM¥¥ } \\
\text { com material } \\
\text { R\$ }\end{array}$ & 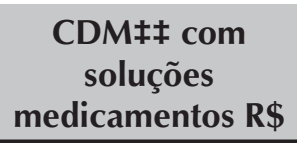 & $\begin{array}{l}\text { CDMT§§ } \\
\text { R\$ }\end{array}$ & $\%$ \\
\hline $\begin{array}{l}1^{a} \text { Preparo da máquina e do circuito } \\
\text { extracorpóreo }\end{array}$ & 14,24 & 84,04 & 22,68 & 120,96 & 27,82 \\
\hline $2^{a}$ Instalação & 6,81 & 13,12 & 8,63 & 28,56 & 6,57 \\
\hline $\begin{array}{l}3^{a} \text { Administração de soluções/ } \\
\text { medicamentos }\end{array}$ & - & 1,71 & 9,08 & 10,79 & 2,48 \\
\hline $4^{\text {a }}$ Desinstalação & 6,72 & 0,9 & 1,17 & 8,79 & 2,02 \\
\hline $5^{\mathrm{a}}$ Monitorização & 205,58 & - & - & 205,58 & 47,28 \\
\hline $\begin{array}{l}6^{a} \text { Desinfecção interna e limpeza } \\
\text { externa da máquina }\end{array}$ & 33,24 & 0,33 & 8,53 & 42,1 & 9,68 \\
\hline $7^{\mathrm{a}}$ Tempo total de deslocamento & 18,05 & - & - & 18,05 & 4,15 \\
\hline CDMT§§ do procedimento HDC†† & 284,64 & 100,1 & 50,09 & 434,83 & 100 \\
\hline
\end{tabular}

extracorpóreo", R\$120,96 (27,82\%) e "desinfecção interna e limpeza externa da máquina", R\$ 42,10 $(9,68 \%)$ na composição do CDMT da HDC (R\$ 434,83).

Reitera-se que na "monitorização" contabilizou-se unicamente a MOD/minuto (R\$ $0,84)$ do técnico de enfermagem do $C D$, que permaneceu ao lado do paciente por um tempo médio de 244,73 minutos, representando $72,69 \%$ do CDM com pessoal.

No "preparo da máquina e do circuito extracorpóreo", o elevado CDM decorreu dos custos com material ( $\mathrm{R} \$ 84,04$ - 69,48\%), especificamente pelos custos unitários dos componentes do circuito extracorpóreo (linha arterial/R\$ 6,30, dialisador capilar/R \$66,72, linha venosa/R $\$ 7,20)$, que foram descartados após o uso, seguido dos custos com soluções/medicamentos ( $R \$ 22,68-18,75 \%$ ), com destaque para os galões de solução de bicarbonato de sódio 8,4\% (5 litros/ $\mathrm{R} \$ 8,30$ ) e de solução polieletrolítica (5 litros/R\$ $9,34)$.

O CDM da etapa "desinfecção interna e limpeza externa da máquina" foi determinado pelo custo com MOD do técnico de enfermagem, $11,68 \%$ do custo com pessoal, seguido do custo com soluções/medicamentos especificamente relacionado ao uso de peróxido de hidrogênio $(300 \mathrm{ml} / \mathrm{R} \$ 8,28)$ empregado para a desinfecção interna da máquina.

$\mathrm{Na}$ composição do CDM da "instalação", o maior custo se concentrou nos materiais, $\mathrm{R} \$$ 13,12 , sendo os custos mais altos relativos ao uso de conectores de proteção das vias do CDL ( $R$ \$ 10,00/par) e ao curativo filme transparente (R\$ 2,97/unidade) e nos medicamentos/soluções, R\$ 7,35 , pelo uso de heparina $(R \$ 1,31 / \mathrm{ml})$, solução fisiológica $\quad 0,9 \% \quad$ (R\$ $0,10 /$ frasco-ampola) $\quad \mathrm{e}$ aplicação de pomada mupirocina ( $\mathrm{R} \$ 0,58 / 10 \mathrm{mg}$ ) para curativo do local de inserção do CDL.

Durante a realização da HDC, o CDM da etapa "administração de soluções e medicamentos" foi determinado pela prescrição do acréscimo de ampolas de cloreto de potássio (R\$ 0,12/ampola), no galão de solução polieletrolítica, variando de cinco a 16, com maior frequência de oito ampolas, e infusão de SF 0,9\% (R\$ 1,90/frasco1000 $\mathrm{ml}$ ) para lavagem do circuito extracorpóreo no caso de pacientes com contra-indicação de anticoagulação sistêmica com heparina.

O CDM das etapas "desinstalação" e "tempo total de deslocamento do técnico de enfermagem" foi condicionado pelos custos com pessoal, R\$ $6,72(76,45 \%)$ e $R \$ 18,05(100 \%)$.

\section{DISCUSSÃO}

Muitos países reconhecem que serviços de saúde são caros e, consequentemente, os gastos em saúde são altos(15-16). Nas organizações hospitalares, a elevação crescente dos custos, para o atendimento das diferentes necessidades de cuidados, demanda a realização de estudos sobre os aspectos financeiros a fim de propiciar estratégias que contribuam com o uso racional dos recursos, equilibrando a oferta de serviços de saúde e a sua viabilidade econômica(17-18).

O aumento dos gastos e dos custos em saúde com o emprego de uma das modalidades 
de TRS, frequentemente a hemodiálise, vem acompanhando as alterações demográficas populacionais, como a tendência de envelhecimento e a mudança do perfil epidemiológico, com o aumento da prevalência de doenças crônico-degenerativas, principalmente a hipertensão e o diabetes ${ }^{(19)}$. A tomada de decisão no campo assistencial, como a escolha de um ou outro procedimento dialítico, depende de questões financeiras e de ressarcimento por parte do Estado, logo esta decisão está relacionada aos recursos disponíveis, bem como com a avaliação do benefício gerado ${ }^{(20)}$.

O CDMT da HDC correspondeu a R $\$ 434,83$ $(\mathrm{DP}=65,10)$, tendo variado entre $\mathrm{R} \$ 308,93$ a $\mathrm{R} \$ 741,48$. Chama a atenção que o valor médio obtido é 1,64 maior do que o valor estabelecido pelo MS, $\mathrm{R} \$ 265,41$, por sessão de hemodiálise realizada a pacientes renais agudos/crônicos agudizados, sem tratamento dialítico iniciado(10), valor reajustado em novembro de 2013, indicando a necessidade de negociação para revisão do repasse do MS ao HCE. Ressalta-se que o CDMT seria mais elevado caso tivesse sido possível a identificação dos custos indiretos, necessários à apuração do custo total(14) da HDC, como exemplos, os custos relativos ao consumo de água e de energia elétrica, horas de manutenção preventiva e corretiva da máquina de HDC e da osmose reversa.

Pesquisas ${ }^{(17,21-22)}$ sobre custos diretos de procedimentos conduzidos por profissionais de enfermagem em outras organizações de saúde, evidenciaram também dificuldades de acesso a informações que fundamentassem o cálculo dos custos indiretos relacionando-as à inexistência de um mecanismo formal para recolher, organizar e comunicar informações contábeis ${ }^{(23)}$.

Apesar da apuração do custo total da HDC não ter se constituído em foco deste estudo, considera-se que a análise dos seus custos diretos contribui com a geração de conhecimentos que podem favorecer, ao HCE, a alocação eficiente dos recursos requeridos. Ademais, a metodologia proposta representa um avanço na obtenção de informações econômicas que propiciem a negociação entre os hospitais públicos de ensino e pesquisa, contratualizados junto ao SUS, e o gestor estadual ou municipal para o ajuste do repasse financeiro.

Tais ajustes se fazem necessários, notadamente quando se tratam de procedimentos cujos valores, mesmo após reajustes, não acompanham a inflação intrínseca do setor saúde, cujos preços, segundo dados do Instituto de Estudos de Saúde Suplementar, crescem mais do que os da economia em geral ${ }^{(24)}$. Negociações desta natureza são viáveis, visto que legalmente é permitida ao gestor a adoção de valores diferenciados aos da Tabela de Procedimentos do SUS, mas não abaixo dos valores por ela determinados, para remuneração dos serviços de saúde, desde que a diferença seja complementada com recursos próprios, estaduais e/ou municipais ${ }^{(25)}$.

Diferentemente dos achados de outras pesquisas nas quais prevaleceram os custos com materiais, soluções/medicamentos frente aos custos com pessoal ${ }^{(17,21-22)}$, o CDM com a MOD do técnico de enfermagem, que correspondeu a $65,04 \%$ do CDMT da HDC, era esperado neste estudo. Isso porque, no Brasil, as normas legais em vigor determinam a presença de profissionais especializados que são responsabilizados pelo atendimento que se fizer necessário durante o procedimento hemodialítico ${ }^{(9)}$.

Então, a participação dos técnicos de enfermagem, especialmente na "monitorização das sessões de HDC", é imprescindível uma vez que estes devem manter observação constante dos sinais e sintomas apresentados pelo paciente, monitorar o funcionamento dos materiais $\mathrm{e}$ equipamentos utilizados, tomar decisões e agir prontamente visando à resolução de ocorrências ou minimização de suas consequências ${ }^{(8)}$.

Oconhecimentodos custos dos procedimentos é vital para estimativas futuras e constituise na base do processo de orçamentação e financiamento de cada unidade nas instituições de saúde ${ }^{(26)}$.

Nessa direção, as organizações hospitalares precisam de informações confiáveis sobre os custos incorridos na prestação dos seus serviços para auxiliá-las na aplicação dos recursos escassos, notadamente em hospitais públicos financiados pelo SUS. Portanto, concorda-se que os enfermeiros podem melhorar o desempenho dessas organizações ao se apropriarem de métodos de custeio, identificando e monitorando os elementos e as etapas do processo assistencial que aumentam os custos e cuja eliminação não traga prejuízos à qualidade ${ }^{(27)}$.

\section{CONCLUSÕES}

O CDMT do procedimento HDC correspondeu a $R \$ 434,83(D P=65,10)$ sendo o CDM das etapas "monitorização" (R\$ 205,58), "preparo da máquina e do circuito extracorpóreo" (R\$ 120,96) 
e "desinfecção interna e limpeza da máquina" (R\$ $42,10)$ os mais importantes na sua composição.

Espera-se que os resultados obtidos estimulem o desenvolvimento de estudos semelhantes em outras UTIs, de organizações públicas e privadas, visto que a metodologia proposta poderá fundamentar a tomada de decisões relativas ao incremento dos recursos envolvidos na consecução da HDC realizada a pacientes portadores de LRA.

Diante da ausência e/ou dificuldade de acesso a informações que possibilitassem, também, a apuração dos custos indiretos, que comporiam o custo total da HDC, este estudo se restringiu aos custos diretos. Então, estudos subsequentes precisariam superar os desafios presentes na identificação dos custos indiretos para apuração dos custos totais da HDC, pois, desta forma, obterse-ão subsídios para contribuir com a melhor gestão econômica e financeira desta modalidade de TRS.

\section{REFERÊNCIAS}

1. Sesso RCC, Lopes AA, Thomé FS, Lugon JR, Burdmann EA. Censo Brasileiro de Diálise, 2009. J. Bras. Nefrol. [Internet] 2010; 32(4) [acesso em 08 jul 2015]. Disponível: http://dx.doi.org/10.1590/S010128002010000400007.

2. National Institute of Diabetes and Digestive and Kidney Diseases. Treatment Methods for Kidney Failure. U.S. [Internet] Centers for Disease Control and Prevention [acesso em 03 jun 2015]. Disponível: http:// kidney.niddk.nih.gov/kudiseases/pubs/hemodialysis/.

3. Liu FX, Walton SM, Leipold R, Isbell D, Golper TA. Financial implications to Medicare from changing the dialysis modality mix under the bundled prospective payment system. Perit Dial Int. [Internet] 2014; 34(7) [acesso em 05 jun 2015]. Disponível: http://pdiconnect. com/content/early/2014/09/30/pdi.2013.00305. abstract.

4. Kleophas W, Reichel H. International study of health care organization and financing: development of renal replacement therapy in Germany. Int J Health Care Finance Econ. 2007; 7(2-3):185-200.

5. Ricci Z, Ronco C, D'Amico G, De Felice R, Rossi S, Bolgan I, et al. Practice patterns in the management of acute renal failure in the critically ill patient: an international survey. Nephrol Dial Transplant. 2006; 21(3):690-6.

6. Davenport A. Renal replacement therapy in acute kidney injury: which method to use in the intensive care unit?. Saudi J Kidney Dis Transpl. 2008; 19(4):529-
36.

7. Klarenbach SW, Tonelli M, Chui B, Manns BJ. Economic evaluation of dialysis therapies. Nat Rev Nephrol. 2014; 10(11):644-52.

8. Lima AFC, Fuzii SMO, Pinho NA, Melo ACT, Hashimoto THF. Processo de enfermagem na prática de hemodiálise: a experiência das enfermeiras de um Hospital Universitário. Revista Referência. 2010; 2(12):39-45.

9. Ministério da Saúde (BR). Agência Nacional de Vigilância Sanitária. Diretoria Colegiada. ResoluçãoRDC $\mathrm{n}^{\circ} 11$ de 13 de março de 2014. Dispõe sobre os requisitos de boas práticas de funcionamento para os serviços de diálise e dá outras providências. Diário Oficial da União, [Internet] 14 mar 2014 [acesso em 08 jul 2015]. Disponível: http://portal.anvisa.gov.br/wps/ wcm/connect/32cb310043da93a4969197937783f3a1/ rdc0011_13_03_2014.pdf?MOD=AJPERES.

10. Ministério da Saúde (BR). Portaria $\mathrm{n}^{\circ}$ 1.331/GM de 27 de novembro de 2013. Altera valores de remuneração e inclui procedimentos de Terapia Renal Substitutiva na Tabela de Procedimentos, Medicamentos, Órteses, Próteses e Materiais Especiais do Sistema Único de Saúde (SUS). Diário Oficial da União, [Internet] 12 dez 2013 [acesso em 08 jul 2015]. Disponível: http://bvsms.saude.gov.br/bvs/saudelegis/sas/2013/ prt1331_27_11_2013.html.

11. Ministério da Saúde (BR). Assessoria de Comunicação. Entendendo o SUS. Brasília: Ministério da Saúde; 2006.

12. Dallora MELV, Forster AC. A importância da gestão de custos em hospitais de ensino - Considerações Teóricas. Medicina (Ribeirão Preto). 2008; 41(2):135-42.

13. Yin RK. Estudo de caso: Planejamento e Métodos. Trad. de Ana Thorell. $5^{a}$ ed. Porto Alegre: Bookman; 2015.

14. Martins E. Contabilidade de custos. $10^{\mathrm{a}}$ ed. São Paulo: Atlas; 2010.

15. Unger F. Health is wealth: considerations to european healthcare. Prilozi. 2012; 33(1):9-14.

16. Sagan A, Panteli D, Borkowski W, Dmowski M, Domanski F, Czyzewski M, et al. Poland health system review. Health Syst Transit. 2011; 13(8):1-193.

17. Nobrega CR, Lima AFC. Procedures' costs related to outpatient chemotherapy treatment of women suffering from breast cancer. Rev. esc. enferm. USP. [Internet] 2014;48(4) [acesso em 08 jul 2015] Disponível: http://dx.doi.org/10.1590/S0080-623420140000400018.

18. Lopes LA, Dyniewicz AM, Kalinowski LC. Gerenciamento de materiais e custos hospitalares 
em UTI Neonatal. Cogitare Enferm. [Internet] 2010; 15(2) [acesso em 08 jul 2015] Disponível: http://dx.doi. org/10.5380/ce.v15i2.17862.

19. Sancho LG, Dain S. Análise de custo-efetividade em relação às terapias renais substitutivas: como pensar estudos em relação a essas intervenções no Brasil?. Cad. Saúde Pública. 2008; 24(6):1279-90.

20. Klarenbach S, Manns B. Economic evaluation of dialysis therapies. Semin Nephrol. 2009; 29(5):524-32.

21. Lima AFC, Castilho V, Fugulin FMT, Silva B, Ramin NS, Melo TO. Costs of most frequent nursing activities in highly dependent hospitalized patients. Rev LatinoAm Enfermagem. [Internet] 2012; 20(5) [acesso em 08 jul 2015] Disponível: http://dx.doi.org/10.1590/S010411692012000500009.

22. Gouveia AL, Lima AFC. Direct cost of connecting, maintaining and disconnecting patient-controlled analgesia pump. Rev. esc. enferm. USP. [Internet] 2014; 48(1) [acesso em 08 jul 2015]. Disponível: http://dx.doi. org/10.1590/S0080-623420140000100013.

23. Bonacim CAG, Araujo AMP. Gestão de custos aplicada a hospitais universitários públicos: a experiência do Hospital das Clínicas da Faculdade de Medicina de Ribeirão Preto da USP. Rev. Adm. Pública. 2010; 44(4):903-31.

24. Instituto de Estudos de Saúde Suplementar. Saúde suplementar frente às demandas de um mundo em transformação. Série IESS 001/2006. São Paulo: IESS; 2006.

25. Ministério da Saúde (BR). Ministério da Educação. Portaria Interministerial $n^{\circ} 1006$, de 27 de maio de 2004. Diário Oficial da União, [Internet] 29 out 2004 [acesso em 08 jul 2015]. Disponível: http://sistema4.saude. sp.gov.br/sahe/documento/portaria/PI_1006_270504. pdf.

26. Gonçalves MA, Zac JI, Amorim CA. Gestão estratégica hospitalar: aplicação de custos na saúde. R. Adm. FACES Journal. 2009; 8(4):161-79.

27. Castilho V, Fugulin FMT, Gaidzinski RR. Gerenciamiento de costos en los servicios de enfermería. In: Kurcgant $\mathrm{P}$, organizadora. Gerenciamiento en enfermería. $2^{a}$ ed. Rio de Janeiro: Guanabara Koogan; 2010. p. 169-80. 